\title{
卫星跟踪技术在候鸟迁徙研究中的应用
}

\author{
伍和启 ${ }^{1,2}$, 杨晓君 ${ }^{1, *}$, 杨君兴 ${ }^{1}$ \\ (1. 中国科学院昆明动物研究所, 云南 昆明 650223；2. 中国科学院研究生院, 北京 100049)
}

\begin{abstract}
摘要: 自 20 世纪 80 年代末期应用基于 Argos 系统的卫星跟踪技术开展候鸟迁徙研究以来, 鸟类学家取得了 许多利用传统鸟类迁徙研究方法所无法取得的成果。但在中国, 卫星跟踪技术在鸟类迁徙中的应用尚属空白。作 者利用参与中国首次应用卫星跟踪技术跟踪候鸟迁徙研究之机, 对该技术用于候鸟迁徙研究的技术平台、主要内 容和未来研究进行了综述和展望。利用卫星跟踪技术开展的主要研究内容有: 揭示迁徙路线和重要停歇地点; 寻 找新繁殖地和越冬地; 利用卫星数据对栖息地及其利用进行评价; 探讨鸟类的迁徙策略。期望该技术能够成为中 国濒危鸟类保护的有效方法, 并尽快得到应用。
\end{abstract}

关键词: Argos 系统; 卫星跟踪技术; 候鸟; 迁徙; 保护

中图分类号: Q959.7; Q958.13 文献标识码: A 文章编号: 0254-5853-(2008)03-0346-07

\section{Review of Satellite Tracking in Bird Migratory Studies}

\author{
WU He-qi ${ }^{1,2}$, YANG Xiao-jun ${ }^{1, *}$, YANG Jun-xing ${ }^{1}$ \\ (1. Kunming Institute of Zoology, the Chinese Academy of Sciences, Kunming Yunnan 650223, China; \\ 2. Graduate School of Chinese Academy of Sciences, Beijing 100049, P. R. China)
}

\begin{abstract}
Satellite tracking, based on the Argos satellite system, has been used in the studies of migratory birds since the 1980's, and ornithologists have learned a lot using this method. But it is not widely used by Chinese ornithologists. We satellite-tracked several wintering Black-necked Cranes in Yunnan Province, in the winter of 2004, to confirm their migratory routes. We were also able to review the utility, advantage and development of this technology. Recent research has focused on discovering migratory routes and important stopover sites, finding new breeding and wintering areas, evaluating habitat quality and habitat use and discussing migration strategies. We hope this review might promote migratory studies and conservation of birds in China.
\end{abstract}

Key words: Argos system; Satellite tracking; Migratory birds; Migration; Conservation

保护全球候鸟最紧迫的任务是明确鸟类在繁殖 地、中转站、越冬地的生态需求 (Berthold et al, 1991)。了解候鸟的迁徙时间、路线、迁徙途经地 等信息，不但对保护候鸟资源有重要的价值，同时 也可为防止鸟机相撞和预防流行病的传播提供科 学依据。传统研究候鸟迁徙的方法如环志法虽有简 便易行的特点, 但其结果依赖于长周期的监测且回 收效果差, 无法在短期内取得明显结果, 需长期延 续研究 (Zhang \& Yang, 1997)。而卫星跟踪技术具 有跟踪范围尺度广, 时间跨度长, 可以准确地得到 被跟踪对象的迁徙时间、迁徙停留地点以及迁徙路
径等采用常规方法等所无法获得的生物学资料, 可 以在短时间内得到物种移动的大量准确、及时信 息, 并可结合研究对象本身和其依赖环境的特点进 行物种的保护设计 (Seegar, 1996; Woodbridge, 1995)。因此, 自 20 世纪 80 年代末利用人造卫星 技术开展对大型野生动物（包括候鸟）迁徙进行跟 踪研究以来, 极大地促进了候鸟迁徙研究的发展, 在众多物种保护中得到广泛和有针对性的应用, 并 取得了良好的效果 (Gruidae: Kanai et al, 2000; Kanai et al, 2002a, b; Ciconiidae: Higuchi et al, 2000; Shimazaki et al, 2004a, b; Threskiornithidae: Ueta et

收稿日期：2008-01-25; 接受日期：2008-03-06

“通讯作者 (Corresponding author), E-mail: yangxj@mail.kiz.ac.cn 第一作者 E-mail: wuhq01@mail.kiz.ac.cn 
al, 2002; Falconiformes: B-U Meyburg \& Meyburg, 2004; Ganusevich et al, 2004; Brodeur et al, 1996; Meyburg et al, 2000; Anseriformes: Kanai et al, 1997; Javed et al, 2000; Gruiformes: Combreau et al, 1999)。同时, 利用卫星遥感技术手段对环境进行 分析的技术日渐成熟, 将卫星跟踪鸟类迁徙和卫星 遥感技术结合进行候鸟栖息地分析并应用到保护 研究方面也已取得很好的结果 (Gratto-Trevor, 1996; Morrison, 1997; Sader et al, 1991; Roughgarden et al, 1991)。

中国的国土跨越了热带、亚热带、温带等自然 气候带; 地形由东部平原逐步上升到西部号称世界 屋脊的青藏高原, 复杂的地形地貌造就了多种类型 的生态环境, 为多达 1244 种的鸟类提供了良好的 繁殖、越冬和停歇环境。我国有候鸟 565 种, 其种 类之多居亚洲各国之首 (Zhang \& Yang, 1997), 同 时我国还位于南北半球鸟类迁徙的主要地带, 在鸟 类迁徙研究中具有重要的地位。我国近代对鸟类迁 徙的研究始于 20 世纪 60 年代, 且仅在个别地方作 过少量研究。全国性的鸟类迁徙研究开始于 80 年 代初期, 但目前仍处于一个相对落后的状态。最近 几年在东南亚爆发的禽流感引发了国内外关于公 共安全的考虑, 迁徙中携带病原微生物的某些候鸟 在疾病传播中扮演了一些角色。中国不但是众多候 鸟的繁殖地或越冬地, 而且还是大量候鸟迁徙的通 道 (这些候鸟迁徙过程中在中国过境或停留)。因 此, 也必将带有潜在的威胁, 清楚、迅速而准确地 了解大型水禽的迁徙路线及其迁徙过程中的信息 对禽流感疾病的传播和防治等涉及公共安全的重 大事件将会有重要意义。这些使得尽快了解和掌握 卫星跟踪技术并用于鸟类迁徙的研究成为急需。在 亚洲、欧洲、北美洲和非洲等地开展的鸟类卫星跟 踪研究为中国鸟类学相关方向研究的开展提供了 很多范例。2004 年冬天, 中国科学院昆明动物研究 所和全国鸟类环志中心等单位首次在中国开展了 利用该技术来跟踪黑颈鹤迁徙的研究, 昆明动物研 究所全程参与了项目的实验设计、野外实施和后期 数据处理等工作。借此机会, 我们综述了该技术在 鸟类迁徙研究中的现状、应用和展望。基于近年来 该技术在保护生物学研究中的迅速发展, 我们希望 本文能够为今后在中国开展同类型研究提供参考。

\section{1 卫星跟踪技术平台}

由卫星发射器 (platform transmitter terminal, 简 称 PTT)、安装在卫星上的传感器、地面接收站三 部分组成的系统构成了该方法的技术平台。其工作 原理是: 卫星上的传感器在接收到由卫星发射器按 照一定间隔发射的卫星信号后, 将此信号传送给地 面接收站处理中心, 经计算机处理后得出跟踪对象 所在地点的经纬度、海拔高度等数据, 提供给研究 者, 研究者最后对获得的数据进行后期处理, 并将 结果用于保护中。

\section{1 卫星发射器}

卫星发射器是安置在研究对象身上的一个主动 式发射系统, 由本身内部携带的电池供电, 以一定 的时间间隔向外界发射信号。为了减少配戴卫星发 射器对研究对象活动的影响, 发射器的重量应该控 制在研究对象体重的 4\%以下 (Higuchi et al，1996, 1998; Kanai et al，1997)。目前开展研究的发射器 基本上是由日本的 NTT、 Toyo Communication Equipment 和美国的 Microwave Telemetry 公司生 产，其中由 Microwave Telemetry, Inc 制造的应用于 鸟类迁徙研究的发射器重量最轻的 $12 \mathrm{~g}$, 最重的为 $95 \mathrm{~g}$ (http://microwavetelemetry.com/index.php)。目 前的发射器都在朝着小型化的方向发展, 研究对象 相应的从大型猛禽、水禽扩大到中等体型的雁鸭 类, 甚至小型的雀形目鸟类等。按照不同的供电方 式, 发射器分为以下 4 种: 以太阳能供电的 Solar PTTs; 以电池供电的 Battery Powered PTTs; 融入 GPS 技术的 Argos/GPS PTTs; 可以植入研究对象体 内的 Implantable PTTs。这些发射器可以配戴在研究 对象的背上、脖子上、脚上和翅膀上等。在鸟类迁 徙的研究中, 大部分使用的是以电池供电的发射 器, 且多采用将发射器绑在鸟类的背部的放置方 法。

发射器固定在研究对象背上的方法 (Fuller et $\mathrm{al}, 1995)$ : 将利用特弗隆可降解材料处理过的细绳 从发射器两端的小孔中穿过, 绳子的两端分别从鸟 右翅膀的前端和左翅膀的后端绕过, 在腹部将绳子 对接, 在接口处沾上胶水、用针线等固定以防脱落, 为防止绳子捆绑过紧影响研究对象的飞行能力, 以 中指和食指的 2-3 指指宽作为参考的松紧度。利 用目前新发展的技术, 脱落的 PTTs 可以被回收再 利用。

\section{2 卫星数据的接收}

研究数据由 Argos 系统提供, 该系统利用美国 
国家海洋和大气局（NOAA）的卫星收集数据。 NOAA 接收、储存 PTT 发射的信号, 然后将信号传 送到地面接收站。地面接收站将接收到的数据进行 初步处理, 计算并转换成可以被研究者直接使用的 经度和纬度坐标, 通过互联网将数据传送给用户。 地理位置被赋予一个数据等级 (location class), 反 映数据的准确性。数据等级分为 7 个级别, 按照准 确度增加的顺序分别是: Z、B、A、0、1、2 和 3 。 按照 Argos 公司 (1992 年) 给出的标准化后实际坐 标的结果, 不同等级所代表的实际坐标经纬度与卫 星提供的经纬度之间有以下的误差范围: A、B 和 Z 级数据的误差范围是 Argos 系统所无法准确估计 的, 0 级数据的误差大于 $1000 \mathrm{~m}, 1$ 级数据的误差 在 $350-1000 \mathrm{~m}, 2$ 级数据的误差在 $150-350 \mathrm{~m}, 3$ 级数据的误差在小于 $150 \mathrm{~m}$ 的范围之内。1-3 级的 数据是高精度的数据, 可以判定研究对象的迁徙、 活动范围以及栖息地利用等。特殊的情况下, 如在 空间和时间上接近跟踪对象的高精度数据的位置 时, $\mathrm{A}$ 和 $\mathrm{B}$ 级的数据可以在判读后被用于确定迁徙 路线当中。

\section{2 在鸟类迁徙和保护中的应用}

现有研究结果主要体现在以下方面: 基本的应 用领域, 监控鸟类在大尺度或全球尺度的运动, 并 评价它们栖息地的特征（Fancy et al, 1988; Priede \& Swift, 1992; Tamura et al, 2000); 深入的研究, 探讨 迁徙机制与迁徙理论的关联, 不同社会状态个体迁 徙行为和机制所表现出的差异等 (Spaar, 1998; Pennycuick et al, 1999; Ueta, 2002; Carlisle et al, 2004; Fujita et al, 2004; Tankersley, 2004)。

\section{1 迁徙路线和重要的停歇地点}

了解物种的具体迁徙路线, 以及迁徙路线的网 络构成和重要的停歇地点对研究对象的保护有不 可替代的作用。由于该技术主要用于候鸟的秋季或 者春季迁徙研究, 因此得出迁徙路线是最为基本和 直接的结果。同时, 该技术能够长时间连续地跟踪 研究对象, 一些重要的迁徙中途停歇地点则能够根 据数据的多少、停留时间的长短等判断和识别。根 据卫星发射器使用时间的长短以及获得的可用数 据的多少, 研究者可以获得不同时间和空间迁徙鸟 类的运动模式。

在短时间内, 如几个月或某个季节内, 研究者 揭示鸟类秋季或者春季的迁徙路线, 或者秋季和春
季两条迁徙路线。这是目前普遍开展的研究内容。 在大型和中等体型的鸟类包括鹤类 (Kanai et al, 2000; Kanai et al, 2002a, b; Higuchi et al, 1996, 1998, 2004; Tamura et al, 2000)、鹳类 (Higuchi et al, 2000; Shimazaki et al, 2004a,b; Ueta et al, 2002)、猛离 (B-U Meyburg \& Meyburg, 2004; Ganusevich et al, 2004; Brodeur et al, 1996; Meyburg et al, 2000)、雁鸭 类（Kanai et al, 1997; Javed et al, 2000）以及大铇 (Otis tarda) (Combreau et al, 1999) 的研究中, 研 究者都发现了研究对象的迁徙路线, 建立了迁徙鸟 类越冬地和繁殖地之间的关联, 更新了物种和栖息 地保护所需的基本生活史信息。

Serge et al（1996）在金雕（Aquila chrysaetos） 的研究第一次给出了该物种一条覆盖整个完整年 周期的迁徙路线。之后, 德国科学家陆续获得白鹳

(Ciconia ciconia) (Berthod et al, 2002, 2004) 和小 乌雕 (Aquila pomarina) (Meyburg et al, 2004) 一个 和多个年周期的迁徙结果, 更加补充了物种的生活 史。如候鸟对越冬地和繁殖地选择利用的稳定性 (site fidelity) 与否, 越冬地与繁殖地间不同年代 迁徙路线的稳定与变化等。Berthold et al (2004) 利用卫星连续 10 年跟踪了一只白鹳, 发现白鹳在 不同年份之间选择越冬地点、迁徙路线、停歇地点 及其时间存在着变化, 但稳定的越冬地点和迁徙路 线同样存在, 这种变化可能受到食物因素的影响。 Meyburg et al（2004）的工作发现一只雄性小乌雕 在 11 年（1992-2002 年) 间利用同一个筑巢地点, 对繁殖地的选择具有极高的稳定性。

利用迁徙研究获得的卫星数据, 研究者还可以 得到研究对象迁徙开始时间、迁徙速度、迁徙距离、 重要的停歇中转站、新的越冬地和繁殖地等内容。 在东亚 3 种鹤类 [丹顶鹤 (Grus japonensis)、白枕鹤 (Grus vipio）以及白鹤（Grus leucogeranus）]迁徙 的研究中, 丹顶鹤的迁徙距离最短 $(871-2509$ $\mathrm{km})$, 能够不停留的直接从越冬地点飞到繁殖地点; 而白鹤的迁徙距离最长 $(4903-5586 \mathrm{~km})$, 在中途 停歇点也最多, 停歇的时间也长; 白枕鹤迁徙距离 介于两者之间 $(1820-2728 \mathrm{~km}$ ), 尽可能的停留很 短时间 (Siberian Crane: Kanai et al, 2002a, b; White-napped Crane: Higuchi et al, 1996, 2004; Red-crowned Crane: Higuchi et al, 1998; Tamura et al, 2000)。卫星跟踪发现在中国鹳类（Shimazaki et al, 2004a, b; Tamura et al, 2000) 和鹤类 (Higuchi et al, 
1996, 1998, 2000, 2004; Kanai et al, 2000; Tamura et $\mathrm{al}, 2000$ ) 的越冬地点和重要的停留地点包括: 渤海 湾和辽东湾、鄱阳湖、三江平原、天津、齐齐哈尔 白城、黄河三角洲。这些地点目前已经被不同程度 地受到保护，有些地点已经建立了自然保护区。一 些对鹤类和鹳类迁徙保护较为重要却不为我们所 知的地点, 通过对卫星数据的分析, 已经成为保护 的焦点。例如在鄱阳湖, 被卫星跟踪的白鹤（Kanai et al, 2002)、白枕鹤（Higuchi et al, 2004）和东方白 鹳（Shimazaki et al, 2004a) 都在此越冬。由于发现 白头鹤 (Grus monacha) 和白枕鹤 (Higuchi et al, 1996, 2004) 迁徙时在此停留, 黑脸琵路（Platalea minor) (Ueta et al, 2002) 和丹顶鹤 (Higuchi et al, 2000）在此繁殖和越冬, 南北朝鲜之间的军事分界 线区域建立了自然保护区, 以更好的保护鸟类及栖 息地。

\section{2 越冬区域和繁殖区域}

在越冬地和繁殖地开展的研究主要集中在研究 对象的活动中, 如不同季节鸟类的家域变化

(Mcgrady et al, 2003; Wood et al, 2000; Meyburg et al, 2004; Combreau et al, 1999; Ganusevich et al, 2004)。相比于信天翁上万平方公里的活动范围, 大铇数百平方公里的活动面积则显得太小, 也反映 了物种本身生物学特性的差别; 猛禽的活动区域能 够从数百平方公里变化到数千甚至上万平方公里, 这是传统方法所无法观测到的。在繁殖季节同一物 种不同社会状态个体的领域大小的变化, 以及繁殖 对中的雌雄个体活动范围的差异等内容也增加和 补充了物种保护的信息 (Jiguet \& Villarubias, 2004)。这些研究的内容往往受到数据精度、数据 量以及持续时间的影响, 虽然不能够做到生态学和 行为学的系统取样, 但利用这些数据, 结合地理信 息系统的软件工具所包涵的方法, 如最小面积法

(Ganusevich et al, 2004)、核心法 (Wood et al, 2000）等, 还是能够计算出研究对象在不同季节的 活动范围, 进而反映不同状态个体的活动状况。关 于此方面物种保护的例子有两个: Weimerskirch et al（1993）在 1990-1992 年对繁殖期间漂泊信天翁

(Diomedea exulanx) 亲鸟的摄食移动进行了卫星 跟踪, 结果发现在孵卵初期的 14 天, 亲鸟采食的 每天飞行距离是 $1300 \mathrm{~km}$; 在孵卵后期的 10 天内, 平均每天飞行 $400 \mathrm{~km}$ 采食, 而在雉鸟出生后飞行 距离则下降到 $260 \mathrm{~km}$ 。推测导致这种变化的原因
与食物分布、亲鸟和雉鸟所需食物量的差异等因素 有关 (Guan \& Higuchi, 2000)。另一个例子是 Jiguet et al（2004）对黑鹳（Ciconia nigra）的繁殖活动研 究, 通过跟踪两对繁殖个体和 3 个非繁殖个体在繁 殖季节的活动, 作者发现繁殖个体在育雗期利用的 家域面积是 $49556 \mathrm{~km}^{2}$, 在幼鸟离巢后繁殖个体利 用的家域面积是 $48885 \mathrm{~km}^{2}$; 而同时期非繁殖个体 的家域面积分别是 6998 和 $425 \mathrm{~km}^{2}$, 繁殖个体和非 繁殖个体存在着家域重叠的现象; 不同对的繁殖个 体的家域面积在育雉期和离巢后大小相似; 两个家 庭在繁殖成功后的活动范围分别是 53023 和 $112360 \mathrm{~km}^{2}$ 。基于上述结果, 作者建议保护离巢区 半径 $20 \mathrm{~km}$ 内的水质清澈的河网系统, 为黑鹳保护 范围大小的设计提供了依据。

\section{3 栖息地分析}

由于受到数据量、数据精度的限制, 很少有工 作直接利用卫星数据进行研究对象在整个季节内 栖息地偏好和利用变化分析 (Ueta et al, 2003)。Ueta et al 研究了虎头海雕 (Haliaeetus pelagicus) 从春季 迁徙到繁殖季节结束这段时间内利用栖息地变化 情况。栖息地被分为河流、海岸、湖泊和其他四类, 研究者将在每一类型栖息地上卫星数据出现的百 分比作为分析数据, 利用可获得性和实际利用结果 的比较分析, 发现海雕在秋季迁徙开始之前和开始 后对河流的利用率最高; 在越冬季节对海岸线和河 流的利用率较高; 在春季迁徙和度夏时对海岸线区 域利用率最高。这些结果可能与不同时期食物的丰 富度有关联。虽然这种处理方式受到数据准确度的 影响很大, 但他们的工作为今后开展的研究扩展了 方向, 至少, 在数据精度提高和有效数据数量增加 到足够多后，这方面的研究能够大量的开展。

部分研究工作将鸟类迁徙路线中停歇地点的栖 息地状况进行大尺度简单分类，并进行总结分析

(Kanai et al, 2002; Higuchi, 1996, 2004; Shimazaki et al, 2004a; Kanai et al, 1997)。在东亚进行的鹤类 迁徙研究中, 研究者均采用了这些做法。以白枕鹤 为例, 作者将研究对象的经纬度座标, 栖息地类型 (平原、沼泽、海岸沼泽、河流、山川以及农地等) 分别罗列, 并根据数据显示时间、物种的生物学信 息等赋予每个地点一种使用功能, 如休息地点、越 冬地点或繁殖地点等, 并由这些结果遴选出一些重 要的越冬停歇地点或新的越冬地点, 然后进行重要 停歇地点栖息地状况数据收集。基于卫星数据所提 
供的信息, 选择一些在迁徙路线上重要的停歇地点 进行地面栖息地状况的考察和数据收集似乎结果 更好, 但这些做法往往受到迁徙路线的长短、时间 和经费等限制。

将鸟类位置的卫星数据和所在区域的人造卫星 遥感数据迭合分析 (Jiguet et al, 2004; Kanai et al, 1994), 对鸟类的栖息地进行评价或监测, 应该是 物种保护最直接和有效的方法。Kanai et al (1994) 通过将鹤类的迁徙路线覆盖到 Landsat 卫星影像上 来比较繁殖季节成年和幼年白枕鹤在三江平原的 栖息地利用情况, 结果发现成鹤利用沼泽中心部位 且很少离开, 而幼鹤则活动范围很大, 且多在沼泽 的外围。利用 Landsat5 波段的比较结果发现成鹤活 动区域土壤湿度要高于幼鹤活动的区域, 显示成鹤 在繁殖季节利用更为潮湿的栖息地, 而幼鹤利用周 围较为干燥的区域。Higuchi et al (1998) 研究在南 北朝鲜军事分界线区域越冬的 4 只丹顶鹤时, 将鹤 的位置叠加到 Landsat 不同 TM 影像图像上, 发现 2 只鹤白天在该区域内的沼泽和农田内活动, 晚上飞 往附近较远的小山边夜宿; 1 只鹤白天和晚上都在 沼泽和农田内活动; 另一只则白天和夜晚都在小山 边活动。丹顶鹤活动结果的获得对于在此区域形成 有针对性保护措施非常关键。Tamura et al （2000） 将丹顶鹤和东方白鹳迁徙路线和卫星影像迭合, 发 现在俄罗斯两种鸟类偏好于选择在湿地停歇, 而在 中国两种鸟类在农田中停歇较多。造成这些的原因 可能是与中国缺乏湿地有关, 从侧面也反映了两种 鸟对自然环境改变的适应性, 为保护提供了思路。

\section{4 迁徙策略}

这种研究将卫星数据与研究对象的种群信息进 行迭合, 从而比较不同种群或社会状态下迁徙策略 的差异。Ueta \& Higuchi (2002) 分析了白枕鹤的迁 徙路线, 发现在春季迁徙时, 成幼迁徙的策略没有 差异, 如迁徙路线的长度、停歇点之间的距离等, 但在迁徙时间长短和停留时间长短上, 则体现出了 差异, 幼鹤更长。这可能说明幼鹤在迁徙过程中为 了储存更多的脂肪完成迁徙, 需要停留更长的时间 增加取食时间，似乎契合候鸟迁徙的时间、能量最 优并举学说 (Spaar, 1998), 在虎头海雕和黑脸琵 鹭的研究中也发现了相同的趋势。

Pennycuick et al (1999) 给 10 只大天鹅 (Cygnus Cygnus）戴上可以测量外界温度、气压、高度等参 数的发射器, 利用人造卫星分别于 1994、1995、1996
年对它们进行了跟踪，对所得数据结合当时的气象 信息, 利用计算机进行模拟, 发现: 大天我在海上 迁徙时，当太阳或月亮的高度位置与地平线的角度 在一 $4^{\circ}$ 以上，能见度在 $2 \mathrm{~km}$ 以上的条件下，大天 我趋向于横渡大海, 否则就停留在海面上。这一研 究结果在一定程度上支持了鸟类迁徙飞行的外部 定向说（Guan \& Higuchi，2000）。

候鸟的迁徙是否按照最短的适合路线进行是一 个有趣的议题。Fujita et al（2004）利用 5 只白枕鹤 在亚洲的迁徙资料，将鹤迁徙路线上实际被鹤利用 的湿地和草地的面积与最短可能的路线上这些区 域的面积进行比较, 发现鹤在迁徙时更期望选择的 路线包含大面积的湿地和草地, 而不是选择理论上 最短的路线进行。这些说明适合栖息地的存在与否 是影响白枕鹤迁徙路线选择的一个因素。栖息地特 征对迁徙鸟类迁徙路线的影响在除了鹤类以外的 鸟类中也有所发现（Carlisle et al, 2004; Tankersley, 2004)。

\section{3 未来研究的展望}

利用 PTT 进行跟踪候鸟研究的特点之一信号基 本都是由电池供电。电量的限制导致了有效数据很 少，无效数据过多。在信号传输过程中，又容易受 到地形、气候、卫星所在位置等的影响, 更加减少 了高精度数据。虽然在某些研究中研究者将 $1-3$ 级的数据分别计算活动面积发现结果的差异不显 著（Jiguet \& Villarubias, 2004)，一些研究者将数据 精度对物种迁徙速度的影响进行了检验和校正，并 提出了一些转换 (Hays et al, 2001)。但总体来说, 利用 PTT 获得的高精度数据太少，无效数据太多。 影响了该技术的广泛使用。同时，由于大多数发射 器都是由电池供电, 卫星跟踪器的总体重量受技术 瓶颈制约, 尚未完全解决延长供电时间和减少电池 设备重量的矛盾。现在的研究大部分在大型和中型 鸟类中开展。随着电池供电技术的改进, 如利用太 阳能和生物电能作为供电来源, 将会减轻发射器的 重量, 并有望利用到更多的鸟类迁徙研究中。跟踪 器重量减轻、电池使用周期增加等问题解决后, 该 技术有望应用到更多中小型物种的迁徙研究中。

GPS 是一个全球卫星的定位系统, 围绕全球分 布的 24 颗卫星保证了位于世界任一地点的用户都 可以随时接收到至少 4 个导航卫星的信号, 数据准 确性非常高, 误差通常在数十米范围内, 大大高于 
Argos 系统数据的准确度。基于 GPS 技术的跟踪器 已经在某些物种如鸽子的返巢迁徙中得到了运用

(Lipp et al, 2004), 效果非常好。该系统是将一个 类似 GPS 的微型设备安装在研究对象身上, 该设备 内包含有晶片和电池, 前者用来储存数据点, 后者 提供晶片正常工作所需的电力。该系统不同于 PTT, 是一个被动式的接收系统, 工作原理与利用 GPS 接 受卫星信号并确定所在地点的经纬度相同。自设备 启动后一直不停的收集信号并储存在晶片中, 研究 者取回晶片, 导出数据, 作进一步分析。利用该技 术能够得到更多精确的数据点, 但电池使用寿命相 对较短, 且储存卫星数据的晶片必须进行回收。由 于发射器质量小, 可以应用于体型较小的鸟类。对

\section{参考文献：}

Argos Inc. 1992. User Manual[M]. Toulouse: Service Argos.

Berthold P, Terrill SB. 1991. Recent advances in studies of bird migration[J]. Annual Review of Ecological System, 22: 357-378.

Berthold P, Bossche W, Jakubiec Z, Kaatz M, Querner U. 2002. Long-term satellite tracking sheds light upon variable migration strategies of White Storks (Ciconia ciconia) [J]. Journal of Ornithology, 143: 489-495.

Berthold P, Kaatz M, Querner U. 2004. Long-term satellite tracking of White Stork (Ciconia ciconia) migration: Constancy versus variability [J]. Journal of Ornithology, 145: 356-359.

Brodeur S, Bird DM, Fuller M. 1996. Complete migration cycle of Golden Eagles breeding in Northern Quebec [J]. Condor, 98: 293-299.

Carlisle JD, Stock SL, Kaltenecker GS, Swanson DL. 2004. Habitat associations, relative abundance, and species richness of autumn landbird migrants in southwestern Idaho [J]. Condor, 106: 549-566.

Combreau O, Launay F, Bowardi MA. 1999. Outward migration of Houbara Bustards from two breeding areas in Kazakhstan [J]. Condor, 101: 159-164.

Fancy SG, Pank LF, Douglas DC, Curby CH, Garner GW, Amstrup SE, Begelin WL. 1988. Satellite Telemetry, a New Tool for Wildlife Research and Management [M]. Fairbacks: United States Fish and Wildlife Service Resource Publication, 172.

Fujita G, Guan HL, Ueta M, Goroshko O, Krever V, Ozaki K, Mita N, Higuchi H. 2004. Comparing areas of suitable habitats along traveled and possible shortest routes in migration of White-naped Cranes Grus vipio in East Asia [J]. Ibis, 146: 461-474.

Fuller, MR, Seegar, WS, Howey PW. 1995. The use of satellite systems for the study of bird migration [J]. Israel Journal of Zoology, 41: 243-252.

Ganusevich SA, Maechtle TL, Seegar WS, Yates MA, Mcgrady MJ, Fuller M, Schueck LD, Henny CJ. 2004. Autumn migration and wintering areas of Peregrine Falcons Falco peregrinus nesting on the Kola Peninsula, northern Russia [J]. Ibis, 146: 291-297.

Gratto-Trevor CL. 1996. Use of landsat TM imagery in determining important shorebird habitat in the outer Mackenzie Delta, northwest territories [J]. Arctic, 49 (1): 11-22.

Guan HL, Higuchi H. 2000. Review on satellite tracking of migratory birds and its prospect [J]. Zool Res, 21, 412-415. [关鸿亮, 通口广芳. 2000.
野生动物尤其是候鸟来说，回收晶片成为制约该技 术大量推广的一个瓶颈。目前, 数据的回收机制包 括：设备从研究对象身体上自然脱落后回收，通过 接收装置在目标动物附近一定距离内回收; 通过电 话信号或卫星回收等。

针对于野生动物迁徙研究, 上述两种跟踪系统 的使用费用仍然非常昂贵 (一个发射器材料和数据 使用费超过 5000 美元)，且相差不大。未来，相关 制约因素得到改进后，如减轻重量、延长使用时间 以及降低使用费用, 同时, 对数据分析能力和技术 的提高, 基于 Argos 系统和基于 GPS 系统的卫星跟 踪技术应该能够尽快的应用到更广发领域的岛类 迁徙和保护研究中。

卫星跟踪技术在鸟类迁徙研究中的应用和展望. 动物学研究, 21: 412-415.]

Hays GC, Akesson S, Godley BJ, Luschi P, Santidrian P. 2001. The implications of location accuracy for the interpretation of satellite-tracking data [J]. Animal Behaviour, 61: 1035-1040.

Higuchi HO, zaki K, Fujita G, Minton J, Ueta M, Soma M, Mita N. 1996. Satellite tracking of White-naped Crane migration and the importance of the Korean Demilitarized Zone [J]. Conservation Biology, 10: 806-812.

Higuchi H, Shibaev Y, Minton J, Ozaki K, Surmach S, Fujita G, Momose K, Momose Y, Ueta M, Andronov V, Mita N,Kanai Y. 1998. Satellite tracking of the migration of the Red-crowned Crane Grus japonensis [J]. Ecol Res, 13: 273-282.

Higuchi H, Nagendran M, Darman Y, Tamura M, Andronov V, Parilov M, Shimazaki H, Morishita E. 2000. Migration and habitat use of Oriental White Storks from satellite tracking studies [J]. Global Environ Res, 4: 169-182.

Higuchi H, Pierre JP, Krever V, Andronov V, Fujita G, Ozaki K, Goroshko O, Ueta M, Smirensky S, Mita N. 2004. Conservation using remote technologies: Satellite-tracking White-naped Cranes (Grus vipio) in Russia and Asia [J]. Conservation Biology, 18: 136-147.

Javed S, Takekawa JY, Douglas DC, Rahmani AR, Kanai Y, Nagendean M, Choudhury BC, Sharma S. 2000. Tracking the spring migration of a Bar-headed Goose (Anser indicus) across the Himalaya with satellite telemetry [J]. Global Environ Res, 4: 195-205.

Jiguet F, Villarubias S. 2004. Satellite tracking of breeding Black Storks Ciconia nigra: New incomes for spatial conservation issues [J]. Biological Conservation, 120: 153-160.

Kanai Y, Kondoh A, Higuchi H. 1994. Analysis of crane habitat using satellite images [A]. In: Higuchi H, Minton J. The future of cranes and wetlands: Proceedings of the international symposium [C]. Wild Brid Society of Japan, Toyko, 72-85.

Kanai Y, Sato F, Ueta M, Minton J, Higuchi H, Soma M, Mita N, Matsui S. 1997. The migration routes and important restsites of Whooper Swans satellite-tracked from northern Japan [J]. Strix, 15: 1-13.

Kanai Y, Minton J, Nagendran M, Ueta M, Auyrsana B, Goroshko O, Kovhsar A F, Mita N, Suwal RN, Uzawa K, Krever V, Higuchi H. 2000. Migration of demoiselle cranes in Asia based on satellite 
tracking and fieldwork [J]. Global Enviorn Res, 4: 143-153.

Kanai Y, Nagendran M, Ueta M, Markin Y, Rinne J, Sorokin AG, Higuchi H, Archibald GW. 2002a. Discovery of breeding grounds of a Siberian Crane Grus leucogeranus flock that winters in Iran, via satellite telemetry [J]. Bird Conservation International, 12: 327-333.

Kanai Y, Ueta M, Germogenov, N, Negendran M, Mita N, Higuchi H. 2002b. Migration routes and important resting areas of Siberian cranes (Grus leucogeranus) that migrate from north-eastern Siberia and China as revealed by satellite tracking [J]. Biological Conservation, 106: 339-346.

Lipp HP, Vyssotski AL, Wolfer DP,Renaudineau S, Savini M, Troster M, Dell'omo G. 2004. Pigeon homing along highways and exits[J]. Current Biology, 14: 1239-1249.

Mcgrady MJ, Ueta M, Potapov ER, Utekhina I, Masterov V, Ladyguine A, Zykov V, Cibor J, Fuller M, Seegar WS. 2003. Movements by juvenile and immature Steller's Sea Eagles Haliaeetus pelagicus tracked by satellite [J]. Ibis, 145: 318-328.

Meyburg BU, Scheller W, Meyburg C. 2000. Migration and wintering of the Lesser Eagle Aquila pomarina: A study by means of satellite telemetry [J]. Global Environ Res, 4: 183-193.

Meyburg BU, Meyburg C. 2004. Migration, wintering and breeding of a lesser spotted eagle (Aquila pomarina) from Slovakia tracked by satellite [J]. Journal of Ornithology, 145: 1-7.

Morrison RIG. 1997. The use of remote sensing to evaluate shorebird habitats and populations on Prince Charles Island, Foxe Basin, Canada [J]. Arctic, 50 (1): 55-75.

Pennycuick CJ, Bradbury TAM, Einarsson O, Owen M. 1999. Response to weather and light conditions of migrating Whooper Swans Cygnus cygnus and flying height profiles, observed with the Argos satellite system [J]. Ibis, 141: 434-443.

Priede IG, Swift SM. 1992. Wildlife telemetry[A]. Remote Monitoring and Tracking of Animals [M]. London: Ellis Horwood.

Roughgarden J, Running SW, Matson PA. 1991. What does remote sensing do for ecology [J]. Ecology, 72 (6): 1918-1922.

Sader SA, Powell GVN, Rapplle JH. 1991. Migratory bird habitat monitoring through remote sensing [J]. International Journal of Remote Sensing, 12 (3): 363-372.

Seegar WS, Cutchis PN, Fuller MR, Suter JJ, Bhatnagar V, Wall JG. 1996. Fifteen years of satellite tracking development and application to wildlife research and conservation [J]. Johns Hopkins APL Technical Digest, 17 (4): 401-411.

Serge B, Robert D, David MB, Mark F. 1996. Complete migration cycle of Golded Eagles breeding in northern Quebec [J]. Condor, 98: 293-299.

Shimazaki H, Tamura M, Higuchi H. 2004a. Migration routes and important stopover sites of endangered Oriental White Storks (Ciconia boyciana) as revealed by satellite tracking[J]. Memoirs of the National Institute of Polar Research Special Issue, 58: 162-178.

Shimazaki H, Tamura M, Darman Y, Andronov V, Parilov MP, Nagendran M, Higuchi H. 2004b. Network analysis of potential migration routes applied to identification of important stopover sites for Oriental White Sotrk (Ciconia boyciana) [J]. Ecol Res, 19: 683-698.

Spaar R, Stark H, Liechti F. 1998. Migratory flight strategies of Levant Sparrow Hawks: Time of energy minimization [J]. Animal Behaviour, 56: 1185-1197.

Tamura M, Higuchi H, Shimazaki H, et al. 2000. Satellite observation of movements and habitat conditions of Red-crowned Cranes and Oriental White Storks in east Asia [J]. Global Environmental Research, 4: 207-217.

Tankersley RDJr. 2004. Migration of birds as an indicator of broad-scale environmental condition [J]. Environ Monitor Assess, 94: 55-67.

Ueta M, Higuchi H. 2002. Difference in migration pattern between adult and immature birds using satellites [J]. Auk, 119: 832-835.

Ueta M, Mcgrady MJ, Nakagawa H, Sato F, Masterov VN. 2003. Seasonal change in habitat use in Steller's Sea Eagles [J]. Oryx, 37: 110-114.

Weimerskirch H, Salamolard M, Sarrazin F, Jouventin P. 1993. Foraging strategy of wandering albatrosses through the breeding season: A study using satellite telemetry [J]. Auk, 110 (2): 325-342.

Wood AG, Naef-Daenzer B, Prince PA, Croxall JP. 2000. Quantifying habitat use in satellite-tracked pelagic seabirds: Application of kernal estimation to albatross locations [J]. Journal of Avian Biology, 31: 278-286.

Woodbridge B, Finlay KK, Seagar ST. 1995. An investigation of the Swainson's Hawk in Argentina [J]. Journal of Raptor Research, 29: 202-204.

Zhang YF, Yang RL. 1997. Bird Migration Research of China [M]. Beijing: China Forestry Publishing House. [张孚允, 杨若莉. 1997. 中国鸟类 迁徙研究. 北京: 中国林业出版社.]

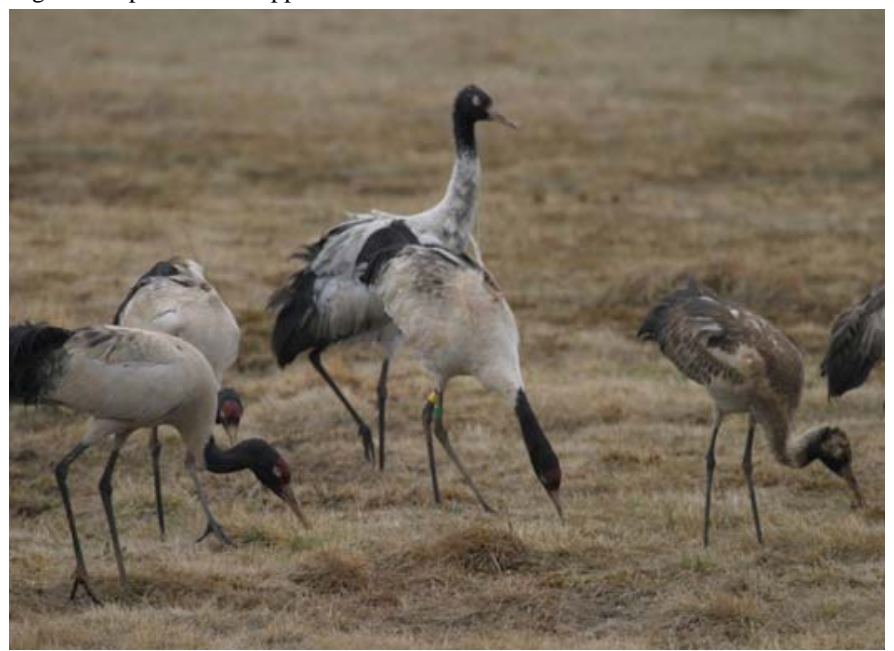

附照 12005 年 3 月 18 日在大山包黑颈鹤国家级自然保护区大海子湿地观察到配戴卫星发射器的黑颈鹤

Black-necked cranes with platform transmitter terminal were observed by satellite tracking at the Dahaizi Wetland in the Dashanbao Black-necked Crane National Nature Reserve on 2005-03-18 